\section{Statistical Properties of the Estimator of the Rotary Coefficient}

\section{S. Chandna and A. T. Walden, Member, IEEE}

\begin{abstract}
Rotary spectral analysis is a widely-used technique for studying elliptical motions in ocean currents, wind, etc. Statistical properties (distribution, bias, confidence intervals) for the estimated rotary coefficient, which measures the tendency to rotate in a counterclockwise or clockwise manner, are derived and applied to ocean current measurements at six depths in the Labrador Sea.
\end{abstract}

Keywords- Complex-valued processes, confidence intervals, probability density function, rotary spectral analysis, rotary coefficient.

\section{INTRODUCTION}

The analysis of two-component real-valued time series, $\left\{X_{t}\right\}$ and $\left\{Y_{t}\right\}$, with power spectra $S_{X}(f)$ and $S_{Y}(f)$ respectively, can be carried out by studying the real-valued vector time series or by considering the complex-valued series $Z_{t}=X_{t}+\mathrm{i} Y_{t}$. The latter approach is widely used in optics, quantum mechanics, electro-magnetics and communications [25], and particularly in physical sciences such as oceanography [6], meteorology [9], [12], [19] and geophysics [2]. The decomposition of the complexvalued series into polarized components, well-summarized in [23], [25], provides the added value of this approach in these sciences; in the frequency domain, the methodology is usually referred to as 'rotary spectral analysis.'

Another major practical advantage is that several useful statistics are invariant to coordinate rotation under the rotary scheme. One of these is the rotary coefficient which we shall study in this article. Consider the following. A zero-mean covariance stationary complex-valued random sequence is harmonizable, so

$$
Z_{t}=\int_{-1 / 2}^{1 / 2} \mathrm{e}^{\mathrm{i} 2 \pi f t} \mathrm{~d} Z(f)
$$

where $Z(f)$ is a random function of $f$ with uncorrelated increments, and zero mean. At frequencies $f$ and $-f$ the contribution to $Z_{t}$ is $\mathrm{d} Z(f) \mathrm{e}^{\mathrm{i} 2 \pi f t}+\mathrm{d} Z(-f) \mathrm{e}^{-\mathrm{i} 2 \pi f t}$ which is the parametric equation of a random ellipse, comprising the addition of two oppositely rotating circular motions [6, pp. 428-9]. The counterclockwise component is considered to be rotating with positive frequency, and vice versa. Depending on which of the two components has the largest magnitude, the complex vector rotates clockwise or counterclockwise with time, with its tip tracing an ellipse. It is then natural to consider the rotary coefficient, defined as [6, p. 431],[7],

$$
\rho(f)=\frac{S_{Z}(f)-S_{Z}(-f)}{S_{Z}(f)+S_{Z}(-f)}
$$

where $S_{Z}(\cdot)$ is the power spectrum for the process $\left\{Z_{t}\right\}$ defined via $E\left\{\mathrm{~d} Z(f) \mathrm{d} Z^{*}\left(f^{\prime}\right)\right\}=\eta\left(f-f^{\prime}\right) S_{Z}(f) \mathrm{d} f$, where $\eta(\cdot)$ is the Dirac delta train with period 1 [4, p. 505]. The rotary coefficient satisfies $-1 \leq \rho(f) \leq 1$ and measures the tendency to rotate in a counterclockwise or clockwise manner; it provides an objective means of quantifying the rotation associated with the

Swati Chandna and Andrew Walden are both at the Department of Mathematics, Imperial College London, 180 Queen's Gate, London SW7 2BZ, UK. e-mail: s.chandna09@imperial.ac.uk and a.walden@imperial.ac.uk asymmetry of the spectrum and is directly related to the expected ellipse shape [25, p. 210]. Let $f>0$. Then if $\rho(f)=+1$, (i.e., $S_{Z}(-f)=0$ ), then motion is all counterclockwise circular at that frequency, whereas if $\rho(f)=-1$, (i.e., $S_{Z}(f)=0$ ), then motion is all clockwise circular at that frequency, and if $\rho(f)=0$, then there is rectilinear motion (unidirectional flow).

To immediately illustrate the very different physical insights gained from the real-valued or complex-valued view of twocomponent time series, we shall make use of ocean current speed and direction time series recorded at a mooring in the Labrador Sea [10], [11]. We associate the eastward (zonal) measurement of current speed with $\left\{X_{t}\right\}$ and the northward (meridional) measurement with $\left\{Y_{t}\right\}$. Fig. 1(a) shows estimated spectra $\hat{S}_{X}(f)$ (thin line) and $\hat{S}_{Y}(f)$ (thick line) for $\left\{X_{t}\right\}$ and $\left\{Y_{t}\right\}$, respectively, at a depth of $110 \mathrm{~m}$. Since the series are real-valued the spectra are symmetric about $f=0$ and only positive frequencies are shown. Fig. 1(b) shows estimated spectra $\hat{S}_{Z}(f)$ (thin line) and $\hat{S}_{Z}(-f)$ (thick line) for positive frequencies. (The spectra are not symmetric in the complex case, and we have folded the negative frequency axis about $f=0$ and overplotted it on the positive frequency axis.) In both plots the vertical dashed line marks the semi-diurnal tidal frequency: the line at this frequency has been estimated and removed ([20, sec. 10.11]) so that the spectra are for the residual current after tide removal. We see that although there are small differences between $\hat{S}_{X}(f)$ (thin line) and $\hat{S}_{Y}(f)$, there is no strong systematic effect, particularly around the semi-diurnal tidal frequency. By way of contrast, using the rotary approach, we see a marked systematic effect: the clockwise spectrum, $\hat{S}_{Z}(-f), f>0$, dominates the counterclockwise spectrum, $\hat{S}_{Z}(f), f>0$, over a band of frequencies around the semi-diurnal tidal frequency - an effect of much interest to oceanographers [6], [7], [21], [26], [29]; we shall return to this data in Section IV.

While there has been widespread use of the rotary coefficient [2], [6], [7], [9], [12], [19], [21], [26], [29], a statistical analysis of its estimator, allowing, for example, the setting of confidence intervals, has not yet been undertaken, and is the purpose of this correspondence.

In Section II we give the background to the construction of the rotary coefficient and the form of its estimator. Section III develops the probability density function (PDF) of the estimator and shows that the associated confidence interval depends on a nuisance parameter; however when this is suitably estimated and debiased the resulting coverage probabilities are very accurate. The forms of the bias and mean squared error of the estimator are also illustrated. In Section IV the statistical methodology is applied to ocean current recordings at six depths in the Labrador Sea, where the local inertial frequency is clearly identified.

\section{BASICS}

\section{A. Background}

Without loss of generality we assume the complex-valued random process $\left\{Z_{t}, t \in \mathbb{Z}\right\}$ to have a mean of zero. Let us define the autocovariance between $Z_{t+\tau}$ and $Z_{t}$ at lag $\tau$ in the usual way as $\operatorname{cov}\left\{Z_{t+\tau}, Z_{t}\right\} \equiv E\left\{Z_{t+\tau} Z_{t}^{*}\right\}$, (where ${ }^{*}$ denotes complex conjugate), and let us also define the relation [22] between $Z_{t+\tau}$ and $Z_{t}$ at lag $\tau$ as $\operatorname{rel}\left\{Z_{t+\tau}, Z_{t}\right\} \equiv$ $E\left\{Z_{t+\tau} Z_{t}\right\}=\operatorname{cov}\left\{Z_{t+\tau}, Z_{t}^{*}\right\} .\left\{Z_{t}\right\}$ is said to be second-order stationary (SOS) iff $\operatorname{cov}\left\{Z_{t+\tau}, Z_{t}\right\}$ and $\operatorname{rel}\left\{Z_{t+\tau}, Z_{t}\right\}$ are functions of $\tau$ only [22], in which case we obtain the autocovariance sequence $\left\{s_{Z, \tau}, \tau \in \mathbb{Z}\right\}$, with $s_{Z, \tau} \equiv \operatorname{cov}\left\{Z_{t+\tau}, Z_{t}\right\}$, and the relation sequence $\left\{r_{Z, \tau}, \tau \in \mathbb{Z}\right\}$, with $r_{Z, \tau}=\operatorname{rel}\left\{Z_{t+\tau}, Z_{t}\right\}$. We note 

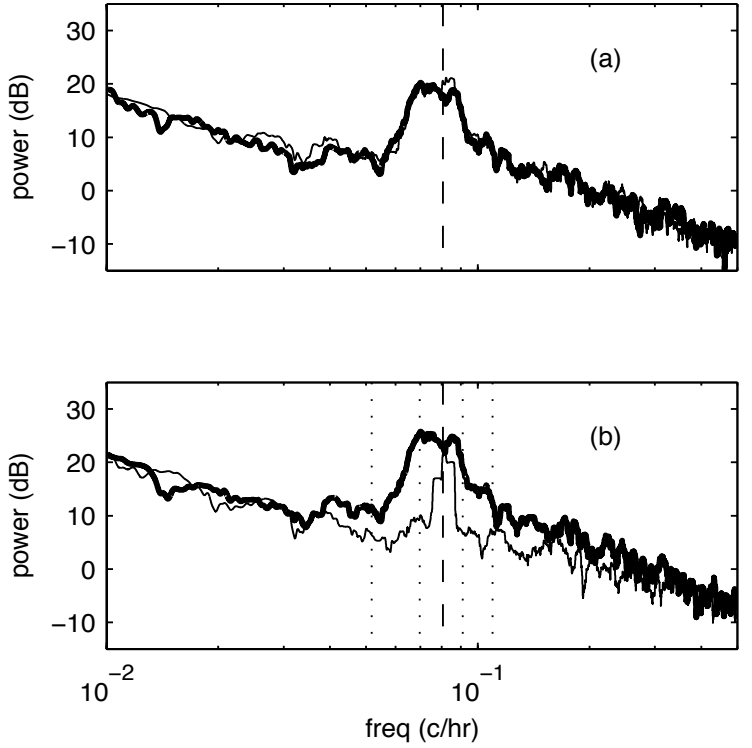

Fig. 1. Analysis of Labrador Sea data for depth of 110m. (a) Estimated spectra of eastward (thin line) and northward (thick line) current velocities. (b) Estimated counterclockwise spectra (thin line) and clockwise spectra (thick line) for complex-valued currents (eastward = real part, northward = imaginary part). For both plots the semi-diurnal tide, (frequency shown by dashed line), has been estimated and removed from the spectra. The role of the vertical dotted lines in plot (b) is explained in the text.

that $s_{Z, \tau}=s_{Z,-\tau}^{*}$, and $r_{Z, \tau}=r_{Z,-\tau}$, i.e., the autocovariance sequence is complex Hermitian, while the relation sequence is complex symmetric.

Let $\left\{\boldsymbol{U}_{t}=\left[Z_{t}, Z_{t}^{*}\right]^{T}, t \in \mathbb{Z}\right\}$, then the Fourier transform $\boldsymbol{S}_{\boldsymbol{U}}(f)$ say, of $\boldsymbol{s}_{\boldsymbol{U}, \tau} \equiv E\left\{\boldsymbol{U}_{t+\tau} \boldsymbol{U}_{t}^{H}\right\}$, is

$$
\begin{aligned}
\boldsymbol{S}_{\boldsymbol{U}}(f) & =\Delta_{\mathrm{t}} \sum_{\tau=-\infty}^{\infty}\left[\begin{array}{ll}
s_{Z, \tau} & r_{Z, \tau} \\
r_{Z, \tau}^{*} & s_{Z, \tau}^{*}
\end{array}\right] \mathrm{e}^{-\mathrm{i} 2 \pi f \tau \Delta_{\mathrm{t}}} \\
& =\left[\begin{array}{cc}
S_{Z}(f) & R_{Z}(f) \\
R_{Z}^{*}(-f) & S_{Z}(-f)
\end{array}\right]
\end{aligned}
$$

where $\Delta_{\mathrm{t}}$ is the sampling interval. The Fourier transform of $\left\{s_{Z, \tau}\right\}$, namely $S_{Z}(f)$, is the power spectrum of $\left\{Z_{t}\right\}$ and is real-valued and non-negative. The Fourier transform of $\left\{r_{Z, \tau}\right\}$, $R_{Z}(f)$, is complex symmetric. Since $r_{Z, \tau}=\operatorname{cov}\left\{Z_{t+\tau}, Z_{t}^{*}\right\}$, $R_{Z}(f)$ is actually the cross-spectrum of $\left\{Z_{t}\right\}$ and $\left\{Z_{t}^{*}\right\}$, and hence we call $R_{Z}(f)$ the relational cross-spectrum. Of course both $S_{Z}(f)$ and $R_{Z}(f)$ are periodic with period unity. The integral of $S_{Z}(f)$ over a period is the process variance, $\sigma_{Z}^{2}$.

Now define $\left\{\boldsymbol{V}_{t}=\left[X_{t}, Y_{t}\right]^{T}, t \in \mathbb{Z}\right\}$. For SOS complex-valued processes the real-valued series $\left\{X_{t}\right\}$ and $\left\{Y_{t}\right\}$ are jointly stationary stochastic processes, and let $s_{\boldsymbol{V}, \tau} \equiv E\left\{\boldsymbol{V}_{t+\tau} \boldsymbol{V}_{t}^{T}\right\}$. Since $\boldsymbol{U}_{t}=\boldsymbol{T} \boldsymbol{V}_{t}$, with

$$
\boldsymbol{T}=\left[\begin{array}{cc}
1 & \mathrm{i} \\
1 & -\mathrm{i}
\end{array}\right]
$$

we have that

$$
\boldsymbol{s}_{U, \tau}=\boldsymbol{T} \boldsymbol{s}_{\boldsymbol{V}, \tau} \boldsymbol{T}^{H}
$$

The spectral matrix for $\left\{\boldsymbol{V}_{t}\right\}$ is given by

$$
\boldsymbol{S}_{\boldsymbol{V}}(f)=\Delta_{\mathrm{t}} \sum_{\tau=-\infty}^{\infty} \boldsymbol{s}_{\boldsymbol{V}, \tau} \mathrm{e}^{-\mathrm{i} 2 \pi f \tau \Delta_{\mathrm{t}}}=\left[\begin{array}{ll}
S_{X X}(f) & S_{X Y}(f) \\
S_{Y X}(f) & S_{Y Y}(f)
\end{array}\right],
$$

where $S_{X X}(f)$ is the spectrum of $\left\{X_{t}\right\}$ and $S_{X Y}(f)$ is the crossspectrum for $\left\{X_{t}\right\}$ and $\left\{Y_{t}\right\}$. So from (4),

$$
\boldsymbol{S}_{\boldsymbol{U}}(f)=\boldsymbol{T} \boldsymbol{S}_{\boldsymbol{V}}(f) \boldsymbol{T}^{H}
$$

from which we find in particular that [25, p. 199]

$$
S_{Z}(f)=S_{X X}(f)+S_{Y Y}(f)+2 \operatorname{Im}\left\{S_{X Y}(f)\right\},
$$

where $\operatorname{Im}\{\cdot\}$ denotes imaginary part.

\section{B. Two Important Properties of the Rotary Coefficient}

Firstly, the rotary coefficient, defined in (2) is invariant to coordinate rotation [17]. To see this note that if we apply a rotation to $\boldsymbol{V}_{t}$ we get

$$
\left[\begin{array}{c}
X_{t}^{\theta} \\
Y_{t}^{\theta}
\end{array}\right]=\left[\begin{array}{cc}
\cos \theta & \sin \theta \\
-\sin \theta & \cos \theta
\end{array}\right]\left[\begin{array}{c}
X_{t} \\
Y_{t}
\end{array}\right]
$$

so that $Z_{t}^{\theta}=X_{t}^{\theta}+\mathrm{i} Y_{t}^{\theta}=Z_{t} \mathrm{e}^{\mathrm{i} \theta}$ and from (1),

$$
Z_{t}^{\theta}=\int_{-1 / 2}^{1 / 2} \mathrm{e}^{\mathrm{i} 2 \pi f t} \mathrm{~d} Z(f) \mathrm{e}^{\mathrm{i} \theta}=\int_{-1 / 2}^{1 / 2} \mathrm{e}^{\mathrm{i} 2 \pi f t} \mathrm{~d} Z^{\theta}(f),
$$

where $\mathrm{d} Z^{\theta}(f)=\mathrm{d} Z(f) \mathrm{e}^{\mathrm{i} \theta}$. Then $E\left\{\left|\mathrm{~d} Z^{\theta}(f)\right|^{2}\right\}=S_{Z}(f) \mathrm{d} f$, and $E\left\{\left|\mathrm{~d} Z^{\theta}(-f)\right|^{2}\right\}=S_{Z}(-f) \mathrm{d} f$, so that the rotary coefficient is unchanged.

Secondly, since $S_{X Y}(-f)=S_{X Y}^{*}(f)$ it follows from (6) that $\rho(f)$ can also be written as

$$
\rho(f)=2 \operatorname{Im}\left\{S_{X Y}(f)\right\} /\left[S_{X X}(f)+S_{Y Y}(f)\right] .
$$

So rectilinear motion, for which $\rho(f)=0$, is equivalent to $\operatorname{Im}\left\{S_{X Y}(f)\right\}=0$, and a test for rectilinear motion at frequency $f$ is equivalent to a test for real structure, for $\boldsymbol{S}_{\boldsymbol{V}}(f)$, i.e., whether $\boldsymbol{S}_{\boldsymbol{V}}(f)=\operatorname{Re}\left\{\boldsymbol{S}_{\boldsymbol{V}}(f)\right\}+\operatorname{iIm}\left\{\boldsymbol{S}_{\boldsymbol{V}}(f)\right\}$ is real-valued. For Gaussian processes the likelihood ratio criterion for testing $H_{0}: \operatorname{Im}\left\{\boldsymbol{S}_{\boldsymbol{V}}(f)\right\}=\mathbf{0}$ against $H_{1}: \operatorname{Im}\left\{\boldsymbol{S}_{\boldsymbol{V}}(f)\right\} \neq \mathbf{0}$ is given by

$$
T_{r}(f)=\operatorname{det}\left\{\hat{\boldsymbol{S}}_{\boldsymbol{V}}(f)\right\} / \operatorname{det}\left\{\operatorname{Re}\left\{\hat{\boldsymbol{S}}_{\boldsymbol{V}}(f)\right\}\right\},
$$

(e.g., [5]), where $\hat{\boldsymbol{S}}_{\boldsymbol{V}}(f)$ estimates $\boldsymbol{S}_{\boldsymbol{V}}(f)$. The distribution of $T_{r}(f)$ is beta with parameters $K-1$ and $1 / 2$, with PDF

$$
f_{T_{r}}(t)=\frac{1}{B(K-1,1 / 2)}(1-t)^{-1 / 2} t^{K-2}, \quad 0<t<1 .
$$

The hypothesis is rejected for too small values of $T_{r}(f)$.

\section{A Rotary Coefficient Estimator}

We use multitaper spectral estimation (e.g., [20]) employing $K$ tapers. We consider tapers which correspond to sampling $K$ rescaled bounded taper functions, each with support on the same finite-length interval of the real line, with the taper functions being orthonormal on this finite interval. The sine tapers (e.g., [28]) used here are of this form.

Form the product $h_{k, t} \boldsymbol{U}_{t}$ of the $t$ th value of the $k$ th real valued taper, $k=0,1, \ldots, K-1$, with the $t$ th value of the sequence, $\boldsymbol{U}_{t}$ and compute the Fourier transform:

$$
\begin{aligned}
\boldsymbol{J}_{\boldsymbol{U}, k}(f) & =\Delta_{\mathrm{t}}{ }^{1 / 2} \sum_{t=0}^{N-1} h_{k, t} \boldsymbol{U}_{t} \mathrm{e}^{-\mathrm{i} 2 \pi f t \Delta_{\mathrm{t}}} \\
& =\boldsymbol{T} \Delta_{\mathrm{t}}{ }^{1 / 2} \sum_{t=0}^{N-1} h_{k, t} \boldsymbol{V}_{t} \mathrm{e}^{-\mathrm{i} 2 \pi f t \Delta_{\mathrm{t}}} \\
& =\boldsymbol{T} \boldsymbol{J}_{\boldsymbol{V}, k}(f)=\left[J_{Z, k}(f), J_{Z, k}^{*}(-f)\right]^{T}
\end{aligned}
$$


where $J_{Z, k}(f)=\Delta_{\mathrm{t}}^{1 / 2} \sum_{t=0}^{N-1} h_{k, t} Z_{t} \mathrm{e}^{-\mathrm{i} 2 \pi f t \Delta_{\mathrm{t}}}$. The multitaper estimator of $\boldsymbol{S}_{\boldsymbol{U}}(f)$ is $[20]$

$$
\hat{\boldsymbol{S}}_{\boldsymbol{U}}(f)=\frac{1}{K} \sum_{k=0}^{K-1} \boldsymbol{J}_{\boldsymbol{U}, k}(f) \boldsymbol{J}_{U, k}^{H}(f)=\left[\begin{array}{cc}
\hat{S}_{Z}(f) & \hat{R}_{Z}(f) \\
\hat{R}_{Z}^{*}(f) & \hat{S}_{Z}(-f)
\end{array}\right] .
$$

The number of tapers, $K$, is the number of complex degrees of freedom of the estimator. Then define,

$$
\hat{A}(f) \equiv \frac{\hat{S}_{Z}(-f)}{\hat{S}_{Z}(f)}=\frac{\sum_{k=0}^{K-1}\left|J_{Z, k}^{*}(-f)\right|^{2}}{\sum_{k=0}^{K-1}\left|J_{Z, k}(f)\right|^{2}} .
$$

The estimator of the rotary coefficient is

$$
\hat{\rho}(f)=\frac{\hat{S}_{Z}(f)-\hat{S}_{Z}(-f)}{\hat{S}_{Z}(f)+\hat{S}_{Z}(-f)}=\frac{1-\hat{A}(f)}{1+\hat{A}(f)} .
$$

We shall also make use of the estimator of the 'conjugate coherence,' i.e., the ordinary coherence between $\left\{Z_{t}\right\}$ and $\left\{Z_{t}^{*}\right\}$ :

$$
\hat{\gamma}_{*}^{2}(f)=\frac{\left|\hat{R}_{Z}(f)\right|^{2}}{\hat{S}_{Z}(f) \hat{S}_{Z}(-f)} .
$$

Assume that $\left\{\boldsymbol{V}_{t}\right\}$ is a bivariate real-valued Gaussian process, then from [4, p. 235], given our taper properties, we can deduce that, asymptotically as $N \rightarrow \infty,\left\{\boldsymbol{J}_{\boldsymbol{V}, k}(f), k=0, \ldots, K-1\right\}$ are distributed independently and identically with a complex bivariate Gaussian distribution with mean $\mathbf{0}$ and covariance matrix $\boldsymbol{S}_{\boldsymbol{V}}(f)$, which we write

$$
\boldsymbol{J}_{\boldsymbol{V}, k}(f) \stackrel{\mathrm{d}}{=} \mathcal{N}_{2}^{C}\left(\mathbf{0}, \boldsymbol{S}_{\boldsymbol{V}}(f)\right), \quad 0<|f|<f_{\mathcal{N}},
$$

where $f_{\mathcal{N}}=1 /\left(2 \Delta_{t}\right)$, the Nyquist frequency. (In practice, the frequency band within which the overall spectral window due to tapering [28] is concentrated, must be narrow enough that the components of $\boldsymbol{S}_{\boldsymbol{V}}(f)$ are essentially constant across it.)

Since $\boldsymbol{J}_{\boldsymbol{U}, k}(f)=\boldsymbol{T} \boldsymbol{J}_{\boldsymbol{V}, k}(f)$, and $\boldsymbol{T} \in \mathbb{C}^{2 \times 2}$, we know that

$$
\boldsymbol{J}_{\boldsymbol{U}, k}(f) \stackrel{\mathrm{d}}{=} \mathcal{N}_{2}^{C}\left(\mathbf{0}, \boldsymbol{T} \boldsymbol{S}_{\boldsymbol{V}}(f) \boldsymbol{T}^{H}\right), \quad 0<|f|<f_{\mathcal{N}}
$$

[1, p. 23], so that, from (5),

$$
\boldsymbol{J}_{\boldsymbol{U}, k}(f) \stackrel{\mathrm{d}}{=} \mathcal{N}_{2}^{C}\left(\mathbf{0}, \boldsymbol{S}_{\boldsymbol{U}}(f)\right), \quad 0<|f|<f_{\mathcal{N}} .
$$

For finite $N$, the independence of the $\boldsymbol{J}_{\boldsymbol{V}, k}(f)$ and the result (17) can only be justified for $W_{N} \leq|f| \leq f_{\mathcal{N}}-W_{N}$, where $\left[-W_{N}, W_{N}\right]$ is the extent of the spectral window induced by tapering [27]; for sine tapers

$$
W_{N}=(K+1) /\left[2(N+1) \Delta_{\mathrm{t}}\right],
$$

(e.g., [28]), which decreases to zero as $N \rightarrow \infty$ for a fixed $K$.

\section{Statistical PRoperties of Rotary COEFficient ESTIMATOR}

\section{A. Distribution of Rotary Coefficient Estimator}

Let $\boldsymbol{W}_{k}=\left[W_{k, 1}, W_{k, 2}\right]^{T}, k=0, \ldots, K-1$, be a size $K$ random sample of two-dimensional complex Gaussian vectors, each $\mathcal{N}_{2}^{C}(\mathbf{0}, \boldsymbol{\Sigma})$. Let $W_{1}=\sum_{k=0}^{K-1}\left|W_{k, 1}\right|^{2}$ and $W_{2}=\sum_{k=0}^{K-1}\left|W_{k, 2}\right|^{2}$. For $0<|f|<f_{\mathcal{N}}$ in the asymptotic case, and $W_{N} \leq|f| \leq$ $f_{\mathcal{N}}-W_{N}$, for finite $N$, the ratio $\hat{A}(f)$ in (12) has the same statistical properties as $W_{2} / W_{1}$, with $\boldsymbol{S}_{U}(f)$ replacing $\boldsymbol{\Sigma}$. Then $[16$, p. 92] the PDF of $\hat{A}(f)$ is given by, for $a \geq 0$,

$$
f_{\hat{A}}(a)=\frac{a^{K-1}(a+q) q^{K}\left(1-\gamma_{*}^{2}\right)^{K}}{B(K, K)\left[(a+q)^{2}-4 a q \gamma_{*}^{2}\right]^{K+(1 / 2)}},
$$

where $B(K, K)=\Gamma^{2}(K) / \Gamma(2 K)$ is the beta function, $q=$ $S_{Z}(-f) / S_{Z}(f)=(1-\rho(f)) /(1+\rho(f))$ is the ratio of the true variances, and $\gamma_{*}^{2}=\left|R_{Z}(f)\right|^{2} /\left[S_{Z}(f) S_{Z}(-f)\right]$ is the conjugate coherence at frequency $f$. (For brevity we have suppressed the frequency dependence of $\hat{A}, q$ and $\gamma_{*}^{2}$ in (19). In what follows we will often suppress explicit frequency dependence where it is understood.) This PDF can be rewritten as

$$
f_{\hat{A}}(a)=\frac{(1+[q / a])(q / a)^{K}\left(1-\gamma_{*}^{2}\right)^{K}}{a B(K, K)\left[(1-[q / a])^{2}+4(q / a)\left(1-\gamma_{*}^{2}\right)\right]^{K+(1 / 2)}} .
$$

Now, $\hat{\rho}=(1-\hat{A}) /(1+\hat{A}) \Rightarrow \hat{A}=(1-\hat{\rho}) /(1+\hat{\rho})$. So, the PDF of $\hat{\rho}$ is

$$
f_{\hat{\rho}}(x)=\left[2 /(1+x)^{2}\right] f_{\hat{A}}([1-x] /[1+x]) .
$$

Then (20) and (21) combine to give

$$
f_{\hat{\rho}}(x)=\frac{2\left(1+y_{\rho x}\right) y_{\rho x}^{K}\left(1-\gamma_{*}^{2}\right)^{K}}{\left(1-x^{2}\right) B(K, K)\left[\left(1-y_{\rho x}\right)^{2}+4 y_{\rho x}\left(1-\gamma_{*}^{2}\right)\right]^{K+(1 / 2)}}
$$

where $y_{\rho x}=[(1-\rho)(1+x)] /[(1+\rho)(1-x)]$, and $|x|,|\rho|, \gamma_{*}^{2}<$ 1. Note that a more complete way of denoting the PDF is $f_{\hat{\rho}}\left(x ; K, \rho, \gamma_{*}^{2}\right)$, which explicitly shows the dependence on the degrees of freedom, $K$, and the two parameters, firstly $\rho$, the true value of the rotary coefficient, and secondly $\gamma_{*}^{2}$, the conjugate coherence. We will sometimes use this longer notation where useful.

Now, let $P(f)$ denote the degree of polarization of the process $\left\{\boldsymbol{U}_{t}\right\}$ (see e.g., [15], [25]). Then, $P^{2}(f)=1-$ $4\left[\operatorname{det}\left\{\boldsymbol{S}_{\boldsymbol{U}}(f)\right\} / \operatorname{tr}^{2}\left\{\boldsymbol{S}_{\boldsymbol{U}}(f)\right\}\right],($ e.g., $[25$, p. 212]) where $\operatorname{det}\{\cdot\}$ and $\operatorname{tr}\{\cdot\}$ denote determinant and trace, respectively. But,

$$
\frac{\operatorname{det}\left\{\boldsymbol{S}_{U}(f)\right\}}{\operatorname{tr}^{2}\left\{\boldsymbol{S}_{\boldsymbol{U}}(f)\right\}}=\frac{S_{Z}(f) S_{Z}(-f)-\left|R_{Z}(f)\right|^{2}}{\left[S_{Z}(f)+S_{Z}(-f)\right]^{2}} .
$$

From this it follows readily that $\left[1-\rho^{2}(f)\right]\left[1-\gamma_{*}^{2}(f)\right]=$ $1-P^{2}(f)$. So given any two of $\rho^{2}(f), \gamma_{*}^{2}(f), P^{2}(f)$, the third is determined. It also follows that $P^{2}(f) \geq \rho^{2}(f)$ and $P^{2}(f) \geq$ $\gamma_{*}^{2}(f)$. Because of these inequalities, and the fact that the true rotary coefficient $\rho(f)$ and the conjugate coherence $\gamma_{*}^{2}(f)$ determine $P^{2}(f)$, we know that $\rho(f)$ and $\gamma_{*}^{2}(f)$ should be chosen as the 'free' parameters for the PDF of the rotary coefficient estimator, $\hat{\rho}(f)$.

Fig. 2 shows the PDF of the rotary coefficient estimator for $K=10$ and different values of $\rho$ and $\gamma_{*}^{2}$. The PDF for negative values of $\rho$ is the reflection around $x=0$ of the PDF for the corresponding absolute value. This can be seen from the fact that when $\rho$ and $x$ are simultaneously negated, $y_{\rho x} \rightarrow 1 / y_{\rho x}$, but $(22)$ is invariant to such an inversion of $y_{\rho x}$, and $\left(1-x^{2}\right)$ in (22) is invariant to a change in the sign of $x$.

\section{B. Confidence Intervals for $\rho(f)$}

Choose a fixed value of $\rho$. If we define points $a_{\alpha / 2}(\rho)$ and $a_{1-\alpha / 2}(\rho)$ such that $F_{\hat{\rho}}\left(a_{\alpha / 2}(\rho) ; K, \rho, \gamma_{*}^{2}\right)=\alpha / 2$ and $F_{\rho}\left(a_{1-\alpha / 2}(\rho) ; K, \rho, \gamma_{*}^{2}\right)=1-\alpha / 2$, where $F_{\hat{\rho}}\left(x ; K, \rho, \gamma_{*}^{2}\right)$ is the distribution function corresponding to the $\operatorname{PDF} f_{\hat{\rho}}\left(x ; K, \rho, \gamma_{*}^{2}\right)$, then

$$
\operatorname{Pr}\left[a_{\alpha / 2}(\rho) \leq \hat{\rho} \leq a_{1-\alpha / 2}(\rho)\right]=1-\alpha .
$$

By taking $\alpha=0.05,95 \%$ confidence regions defined in this way are given in Figs. 3(a) and (b) for $K=10$ and $K=20$, 

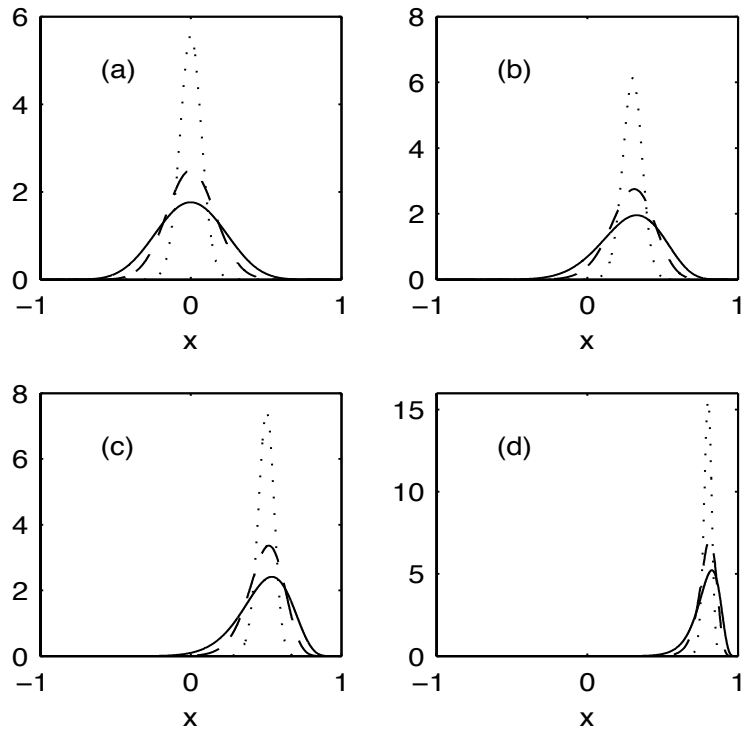

Fig. 2. PDF $f_{\hat{\rho}}\left(x ; K, \rho, \gamma_{*}^{2}\right)$ for $K=10$ and (a) $\rho=0$ (b) $\rho=0.3$ (c) $\rho=0.5$ (d) $\rho=0.8$ using $\gamma_{*}^{2}=0,0.5,0.9$, shown as solid, dashed and dotted lines, respectively.
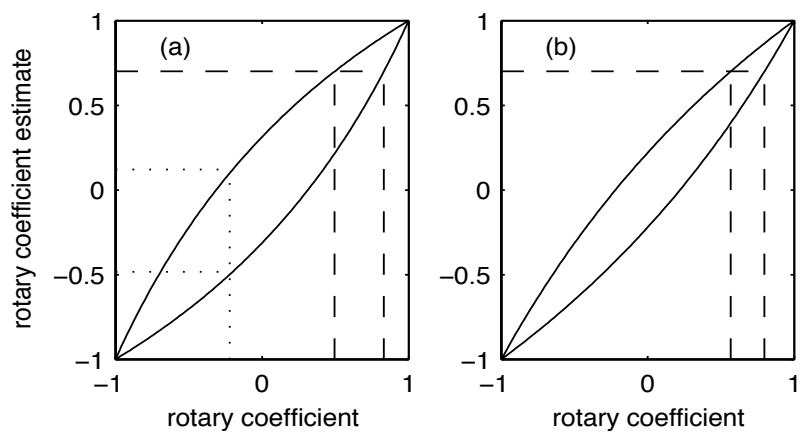

Fig. 3. $95 \%$ confidence regions for $\rho$ when $\gamma_{*}^{2}=0.5$ and (a) $K=10$ and (b) $K=20$. The dashed and dotted lines are explained in the text.

respectively, when $\gamma_{*}^{2}=0.5$. The dotted lines in Fig. 3(a) show the interval (23) for a given value of $\rho$.

Confidence intervals for $\rho$ are found by using the reverse strategy - see the dashed lines in Figs. 3(a) and (b). Given an estimate $\hat{\rho}$, here $\hat{\rho}=0.7$, we draw a horizontal dashed line across the plot at that value; the intersection of this line with $a_{1-\alpha / 2}$ at $\rho_{1}$ say, and with $a_{\alpha / 2}$ at $\rho_{2}$ say, defines a $95 \%$ confidence interval $(\alpha=0.05)$ for $\rho$ as $\left[\rho_{1}, \rho_{2}\right]$, which depends on the $K$ used. The resulting $95 \%$ confidence intervals for $\rho$ are $[0.50$, $0.83]$ for $K=10$ and and $[0.57,0.80]$ for $K=20$ when $\hat{\rho}=0.7$; as expected the interval is narrower with increasing number of complex degrees of freedom $K$.

Denote the $100(1-\alpha) \%$ confidence interval more fully by

$$
\left[\rho_{1}\left(\hat{\rho} ; \alpha, K, \gamma_{*}^{2}\right), \rho_{2}\left(\hat{\rho} ; \alpha, K, \gamma_{*}^{2}\right)\right] .
$$

The interval is random since it depends on $\hat{\rho}$, and it depends on the known quantities $K$ and $\alpha$, and the so-called 'nuisance parameter' $\gamma_{*}^{2}$. Computationally, given an estimate $\hat{\rho}$ of $\rho$, the right end of the interval, $\rho_{2}\left(\hat{\rho} ; \alpha, K, \gamma_{*}^{2}\right)$, is the value of $\rho$ such that $F_{\hat{\rho}}\left(\hat{\rho} ; K, \rho, \gamma_{*}^{2}\right)-\alpha / 2=0$, which can be found simply using any standard zero-finding algorithm. $\rho_{1}\left(\hat{\rho} ; \alpha, K, \gamma_{*}^{2}\right)$ is likewise found by finding the value of $\rho$ for
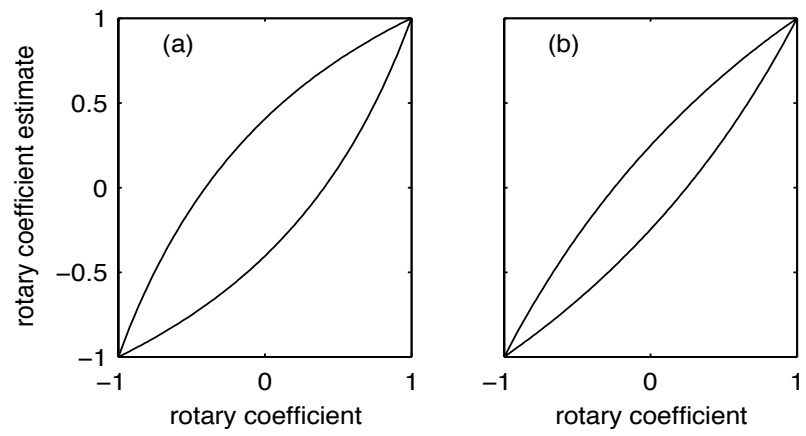

Fig. 4. $95 \%$ confidence regions for $\rho$ when $K=10$ and (a) $\gamma_{*}^{2}=0.1$ and (b) $\gamma_{*}^{2}=0.7$.

which $F_{\hat{\rho}}\left(\hat{\rho} ; K, \rho, \gamma_{*}^{2}\right)-(1-\alpha / 2)=0$. As no analytical form for $F_{\hat{\rho}}\left(\hat{\rho} ; K, \rho, \gamma_{*}^{2}\right)$ is forthcoming, it is necessary to calculate it by numerical integration of the PDF.

Fig. 4 shows the form of the $95 \%$ confidence regions for $K=10$ and $\gamma_{*}^{2}$ values of 0.1 and 0.7 . The region decreases in size with increasing $\gamma_{*}^{2}$. So from Figs. 3 and 4 we see that confidence intervals for $\rho$ narrow as $K$ increases, and also as $\gamma_{*}^{2}$ increases. The latter is explained by the fact that increasing $\gamma_{*}^{2}$ corresponds to increasing $P^{2}$.

\section{Simulated coverage intervals}

The confidence interval (24) assumes knowledge of $\gamma_{*}^{2}$, but in practice this will not be known. We carried out a simulation study to look at the coverage probability when $\gamma_{*}^{2}$ is first estimated, then debiased, and then included in (24) in place of the unknown true value of $\gamma_{*}^{2}$.

Three different matrices playing the role of $\boldsymbol{S}_{\boldsymbol{U}}(f)$ in (3) were used in the simulations:

$$
\boldsymbol{S}_{U}^{(1)}=\left[\begin{array}{cc}
5 & -2+2 \mathrm{i} \\
-2-2 \mathrm{i} & 2
\end{array}\right] ; \boldsymbol{S}_{U}^{(2)}=\left[\begin{array}{cc}
10 & 7+\mathrm{i} \\
7-\mathrm{i} & 10
\end{array}\right]
$$

and

$$
\boldsymbol{S}_{U}^{(3)}=\left[\begin{array}{cc}
2 & 1+\mathrm{i} \\
1-\mathrm{i} & 5
\end{array}\right]
$$

for which $\rho=0.43, \gamma_{*}^{2}=0.8$, for the first, $\rho=0, \gamma_{*}^{2}=0.5$, for the second, and $\rho=-0.43, \gamma_{*}^{2}=0.2$, for the third. For each model matrix we simulated bivariate complex Gaussian vectors as in (17) using the method in $[14$, sec. V] and then combined them as in (11). $K$ was chosen as 10 and 20. For each model matrix 5000 independent realizations of $\hat{\boldsymbol{S}}_{U}$ were produced, and consequently 5000 realizations of $\hat{\rho}$ from (12) and $\hat{\gamma}_{*}^{2}$ from (14). The estimates $\hat{\gamma}_{*}^{2}$ were debiased in two ways. Firstly an unbiased estimate, $\tilde{\gamma}_{*}^{2}$, was obtained as

$$
\tilde{\gamma}_{*}^{2}=1-\left(1-\hat{\gamma}_{*}^{2}\right)_{2} F_{1}\left(1,1 ; K ; 1-\hat{\gamma}_{*}^{2}\right),
$$

(e.g., [13, eqn. 24]), where ${ }_{2} F_{1}(a, b ; c ; y)$ is the hypergeometric function with 2 and 1 parameters and argument $y$. A second debiased estimate, $\bar{\gamma}_{*}^{2}$, was found using the simpler formula [3]

$$
\bar{\gamma}_{*}^{2}=\left[K \hat{\gamma}_{*}^{2}-1\right] /[K-1] .
$$

Since both these debiased estimates can be negative, they must be modified to $\max \left\{0, \tilde{\gamma}_{*}^{2}\right\}$ and $\max \left\{0, \bar{\gamma}_{*}^{2}\right\}$, respectively.

The simulation results are given in Table I. We see that the coverage probabilities closely match the nominal levels whether the exact conjugate coherence is used to define (24), or whether either of the debiased estimates are used. 


\begin{tabular}{|c||c|c|ccc|}
\hline$K$ & Level & Model & \multicolumn{3}{|c|}{ Conj. coherence } \\
& & & $\gamma_{*}^{2}$ & $\tilde{\gamma}_{*}^{2}$ & $\bar{\gamma}_{*}^{2}$ \\
\hline \hline \multirow{2}{*}{10} & $90 \%$ & 1 & 90.1 & 88.3 & 89.8 \\
& & 2 & 89.9 & 88.4 & 89.3 \\
& & 3 & 89.5 & 88.8 & 89.1 \\
\hline \multirow{3}{*}{20} & $95 \%$ & 1 & 95.2 & 94.6 & 95.4 \\
& & 2 & 95.3 & 94.4 & 95.0 \\
& & 3 & 95.3 & 94.8 & 95.0 \\
\hline \hline \multirow{2}{*}{$90 \%$} & 1 & 90.3 & 89.9 & 90.5 \\
& & 2 & 90.1 & 89.3 & 89.6 \\
& & 3 & 90.4 & 89.9 & 90.1 \\
\hline & $95 \%$ & 1 & 95.1 & 94.9 & 95.3 \\
& & 2 & 94.7 & 94.6 & 94.8 \\
& & 3 & 95.1 & 95.2 & 95.3 \\
\hline
\end{tabular}

TABLE I

Percentage of simulated Random intervals containing $\rho$ When CONJUGATE COHERENCE IS KNOWN OR ESTIMATED.
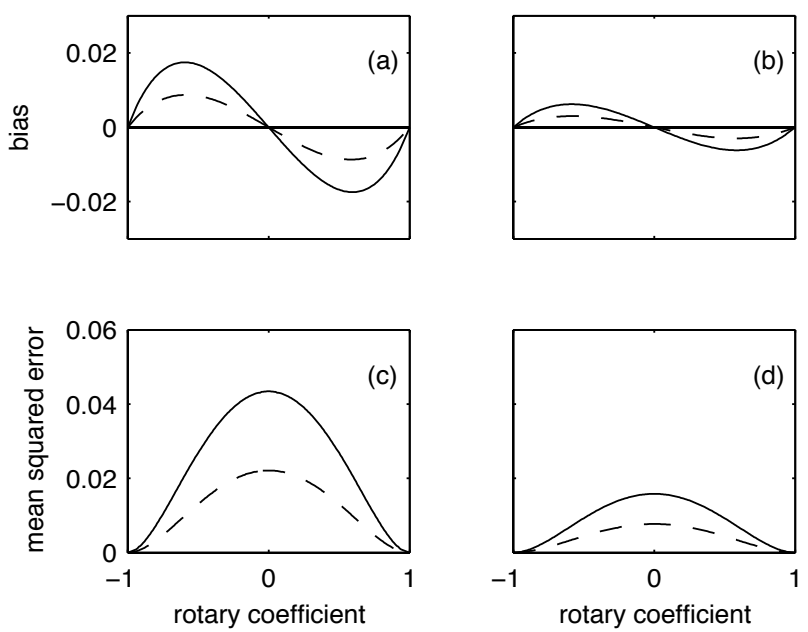

Fig. 5. Bias and mean squared errors (MSE). (a) bias when $\gamma_{*}^{2}=0.1$, (b) bias when $\gamma_{*}^{2}=0.7$, (c) MSE when $\gamma_{*}^{2}=0.1$, and (d) MSE when $\gamma_{*}^{2}=0.7$. Solid lines are for $K=10$ and dashed lines for $K=20$.

The fact that we get close to nominal coverage when the conjugate coherence nuisance parameter is not known but rather is estimated and debiased is the key to making the calculation of confidence intervals for the rotary coefficient of great practical utility in the many scientific studies in which it is routinely used.

\section{Bias and mean squared error}

Two important properties of $\hat{\rho}$, namely the bias, $E\{\hat{\rho}\}-\rho$, and the mean squared error (MSE), $E\left\{(\hat{\rho}-\rho)^{2}\right\}$ (variance plus squared bias), are shown in Fig. 5. Values were readily computed from moments derived from (22) using numerical integration. We see that bias is positive for negative values of $\rho$ and vice versa. Mean squared error is symmetric in $\rho$, and largest at $\rho=0$. Both bias and MSE decrease with increasing $K$ and increasing $\gamma_{*}^{2}$.

\section{Data AnAlysis}

Our example in the Introduction made use of ocean current speed and direction time series for one depth from a set of six
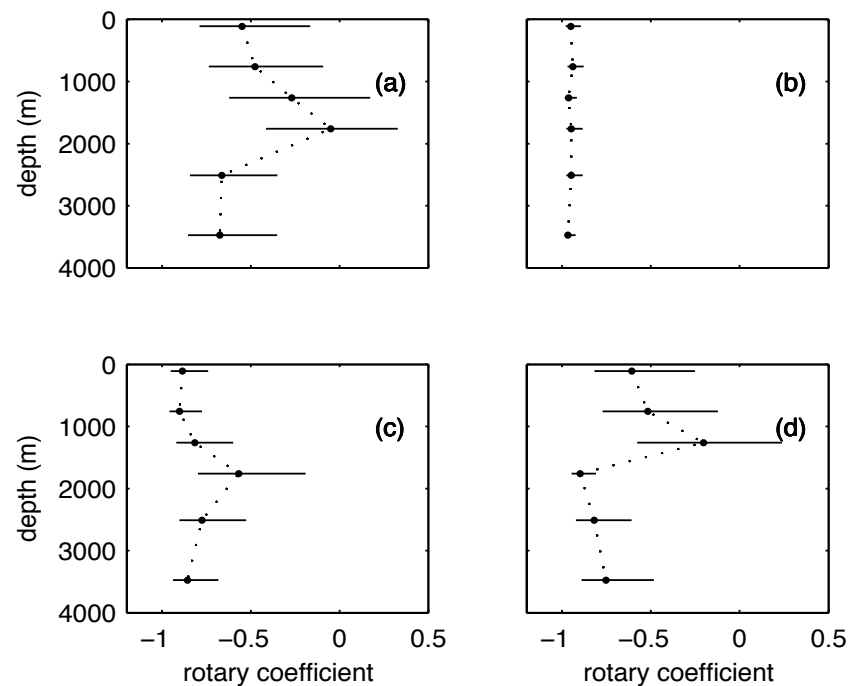

Fig. 6. Estimated rotary coefficient (solid dot) and corresponding 95\% confidence intervals (solid horizontal bars) for the six observation depths at the frequencies (c/hr) (a) 0.0518, (b) 0.0697, (c) 0.0911 and (d) 0.1095. Dotted lines delineate estimated change with depth.

(110, 760, 1260, 1760, 2510 and $3476 \mathrm{~m})$ recorded at a mooring in the Labrador Sea [10], [11]. We used $N=1600$ observations with a sampling interval of $\Delta_{\mathrm{t}}=1 \mathrm{hr}$. In the spectral analysis $K=10$ sine tapers were applied. In this section we shall show the utility of the statistical results in this correspondence in providing insights into the nature of the ocean data. Since $W_{N}$ in $(18)$ is $0.0034 \mathrm{c} / \mathrm{hr}$, the validity range $W_{N} \leq|f| \leq f_{\mathcal{N}}-$ $W_{N}$ for our statistical results for a finite- $N$ sample is given by $0.0034 \leq|f| \leq 0.4966$, hardly different to the asymptotic range $0<|f|<0.5$.

Of great interest to oceanographers are deep ocean motions well away from boundaries, especially in the internal wave frequency band between about $10^{-2}$ and $1 \mathrm{c} / \mathrm{hr}$. Fig. 1 covers this band, fairly central to which is the semi-diurnal tidal frequency (dashed). At a slightly lower frequency than the main tide will be the local inertial frequency which is latitude dependent.

Fig. 6 shows the estimated rotary coefficient (solid dot) and corresponding $95 \%$ confidence intervals (solid horizontal bars) for the six observation depths at the frequencies (c/hr) (a) 0.0518, (b) 0.0697, (c) 0.0911 and (d) 0.1095. These are the frequencies marked by the dotted lines in Fig. 1(b).

Starting with Fig. 6(b) at $f=0.0697 \mathrm{c} / \mathrm{hr}$, we see firstly that here the rotary coefficient is very close to -1 at all depths, (the confidence intervals are very narrow). This is what would be expected as an ideal theoretical outcome at the local inertial frequency, which is thus identified, suggesting that the statistical methodology has performed impressively well. At the frequency $f=0.0911 \mathrm{c} / \mathrm{hr}$, slightly higher than the tidal frequency, the rotary coefficient is more uncertain, Fig. 6(c), but does not appear to deviate greatly from about -0.8 . For the lowest and highest of the four frequencies, Figs. 6(a) and (d) the most notable feature is the large deviations towards 0 at shallow depths.

In Fig. 7(a) we have plotted the estimated rotary coefficient for the depth $760 \mathrm{~m}$, along with $95 \%$ confidence intervals, at a regular (but coarse for clarity) frequency spacing. We notice that the confidence intervals are only narrow for frequencies either side of, but not too near the main tidal frequency. In Fig. 7(b) the heavy bars show the frequency ranges, on a finer frequency grid, at which the null hypothesis of rectilinear flow 

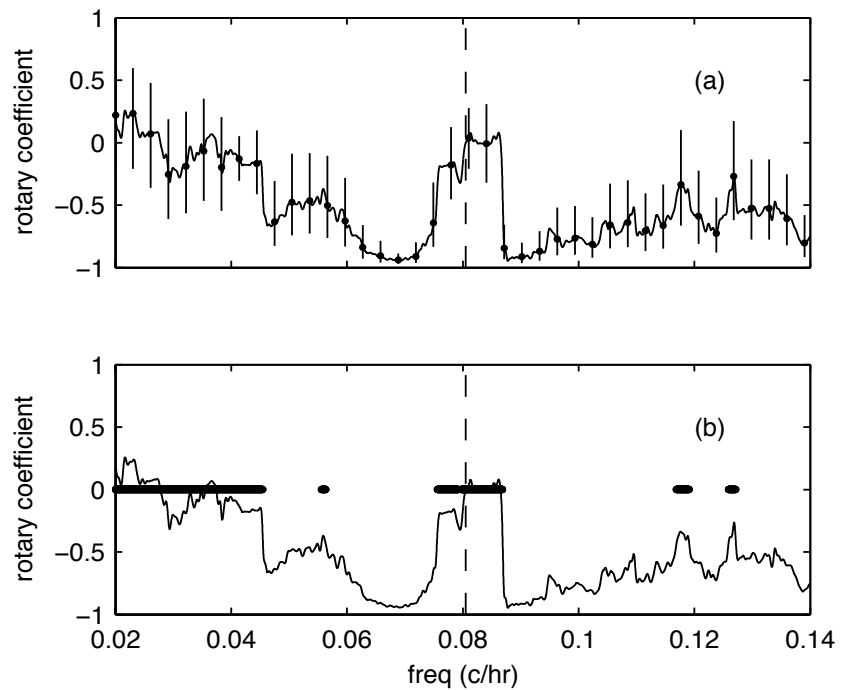

Fig. 7. Variation of estimated rotary coefficient as a function of frequency at depth $760 \mathrm{~m}$. (a) showing $95 \%$ confidence intervals (solid vertical bars) at a regular frequency spacing, (b) showing the frequencies at which the null hypothesis of rectilinear flow is not rejected (heavy bars). The semidiurnal tidal frequency is shown by the vertical dashed line.

is not rejected at the $5 \%$ level. (The statistic (8) was used with distribution (9).) We see that these results are entirely consistent with the confidence intervals in Fig. 7(a); where the confidence interval includes zero the rectilinear flow hypothesis is not rejected, and vice versa.

\section{Concluding COMments}

We have derived the basic statistical properties for the estimated rotary coefficient. These depend on the true value of the rotary coefficient, and the conjugate coherence $\gamma_{*}^{2}$, a nuisance parameter. Fortunately when the latter is estimated and debiased constructed confidence intervals maintain appropriate coverage probabilities, so such confidence intervals have practical utility as illustrated by the Labrador Sea current data analysis.

\section{ACKNOWLEDGEMENT}

The authors are very grateful to Jon Lilly for making the Labrador Sea data available to them. Helpful comments and observations by the reviewers were much appreciated.

\section{REFERENCES}

[1] H. H. Andersen, M. Højbjerre, D. Sørensen and P. S. Eriksen, Linear and Graphical Models. New York: Springer-Verlag, 1995.

[2] C. E. Barton, "Analysis of palaeomagnetic time series - techniques and applications," Surveys in Geophysics, vol. 5, pp. 335-68, 1983

[3] V. A. Benignus, "Estimation of the coherence spectrum and its confidence interval using the fast Fourier transform," IEEE Trans. Audio and Electroac. vol. 17 , pp. 145-50, 1969.

[4] D. R. Brillinger, Time Series: Data Analysis and Theory (Expanded Edition). New York: McGraw-Hill Inc., 1981

[5] E. M. Carter, C. G. Khatri and M. S. Srivastava, "Nonnull distribution of likelihood ratio criterion for reality of covariance matrix," J. Multivariate Analysis, vol. 6, pp. 176-84, 1976.

[6] W. J. Emery and R. E. Thomson, Data Analysis Methods in Physical Oceanography. New York: Pergamon, 1998.

[7] J. Gonella, "A rotary component method for analysing meteorological and oceanographic vector time series," Deep-Sea Research, vol. 19, pp. 833-846, 1972.

[8] I. S. Gradshteyn and I. M. Ryzhik, Table of Integrals, Series, and Products (Corrected and Enlarged Edition). New York: Academic Press, 1980

[9] Y. Hayashi, "Space-time spectral analysis of rotary vector series," J. of the Atmospheric Sciences, vol. 36, pp. 757-66, 1979
[10] J. M. Lilly, P. B. Rhines, M. Visbeck, R. Davis, J. R. Lazier, F. Schott and D. Farmer, "Observing deep convection in the Labrador Sea during winter 1994/95," J. Phys. Oceanogr., vol. 29, 2065-98, 1999.

[11] J. M. Lilly and P. B. Rhines, "Coherent eddies in the Labrador Sea observed from a mooring," J. Phys. Oceanogr., vol. 32, 585-98, 2002.

[12] T. Maitani, "Statistics of wind direction fluctuations in the surface laye over plant canopies," Boundary-Layer Meteorology, vol. 26, 15-24, 1983.

[13] T. Medkour and A. T. Walden, "Attenuation estimation from correlated sequences," IEEE Trans. Signal Process., vol. 55, pp. 378-83, 2007.

[14] T. Medkour and A. T. Walden, "A variance equality test for two correlated complex Gaussian variables with application to spectral power comparison," IEEE Trans. Signal Process., vol. 55, pp. 881-8, 2007.

[15] T. Medkour and A. T. Walden, "Statistical properties of the estimated degree of polarization," IEEE Trans. Signal Process., vol. 56, pp. 408-14, 2008 .

[16] K. S. Miller, Hypothesis Testing with Complex Distributions . New York: Robert E. Krieger, 1980.

[17] C. N. K, Mooers, "A technique for the cross spectrum analysis of pairs of complex-valued time series, with emphasis on properties of polarized components and rotational invariants," Deep-Sea Research, vol. 20, pp. 1129-41, 1973

[18] I. Olkin and J. W. Pratt, "Unbiased estimation of certain correlation coefficients," Annals of Mathematical Statistics, vol. 21, pp. 201-211, 1958.

[19] M. Orlić, B. Penzar, and I. Penzar, "Adriatic sea and land breezes: clockwise versus anticlockwise rotation," J. Appl. Meteorology, vol. 27, pp. 675-9, 1988

[20] D. B. Percival and A. T. Walden, Spectral Analysis for Physical Applications. Cambridge, UK: Cambridge University Press, 1993.

[21] M. S. S. Sarma, and L. V. Gangadhara Rao, "Spectra of currents and temperature off Godavari (East Coast of India)," Mahasagar, vol. 22, pp. 2936,1989 .

[22] B. Picinbono and P. Bondon, "Second-order statistics of complex signals," IEEE Trans. Signal Processing, vol. 45, pp. 411-420, 1997.

[23] P. J. Schreier, "Polarization ellipse analysis of nonstationary random signals," IEEE Trans. Signal Processing, vol. 56, pp. 4330-4339, 2008

[24] P. J. Schreier and L. L. Scharf, "Second-order analysis of improper complex random vectors and processes," IEEE Trans. Signal Processing, vol. 51 pp. $714-725,2003$.

[25] P. J. Schreier and L. L. Scharf, Statistical Signal Processing of ComplexValued Data. Cambridge, UK: Cambridge University Press, 2010.

[26] H. van Haren and C. Millot, "Rectilinear and circular inertial motions in the Western Mediterranean Sea," Deep-Sea Research Part I, vol. 51, pp. $1441-55,2004$.

[27] A. T. Walden, E. J. McCoy and D. B. Percival, "The variance of multitaper spectrum estimates for real Gaussian processes," IEEE Trans. Signal Processing, vol. 42, pp. 479-482, 1994.

[28] A. T. Walden, E. J. McCoy and D. B. Percival, "The effective bandwidth of a multitaper spectral estimator," Biometrika, vol. 82, 201-214, 1995

29] L. Zhiliang, H. Dunxin, T. Xiaohui, and W. Enbo, "Rotary spectrum analysis of tidal current in the southern Yellow sea," Chinese Journal of Oceanology and Limnology, vol. 25, pp. 286-291, 2007. 